\title{
A simple model established by blood markers predicting overall survival after radical resection of type II and III Adenocarcinomas of the Esophagogastric Junction
}

\author{
Li-xiang Zhang \\ Anhui Medical University \\ Zhi-jian Wei \\ Anhui Medical University
}

A-Man Xu ( 18788831253@163.com)

First Affiliated Hospital of Anhui Medical University

\section{Research article}

Keywords: Adenocarcinomas of the esophagogastric junction, neutrophils to lymphocytes ratio, platelets to lymphocytes ratio, cancer, prognosis

Posted Date: January 17th, 2020

DOl: https://doi.org/10.21203/rs.2.21115/v1

License: (c) (1) This work is licensed under a Creative Commons Attribution 4.0 International License.

Read Full License 


\section{Abstract}

\section{Background}

The prognostic prediction after radical resection of Adenocarcinomas of the esophagogastric junction (AEG) has not been well established. We aimed to establish a prognostic model for AEG based on a new score system, which included another clinical routine serum marker.

\section{Methods}

355 patients who underwent curative AEG at the first affiliated Hospital of Anhui Medical University from January 2010 to January 2011 were included in this study. Univariate and multivariate analysis were used to screen for prognostic risk factors. The construction of the nomogram was based on Cox proportional hazard regression models. The construction of the new score models was analyzed by $\mathrm{C}$ index and Calibration curves.

Results

Multivariate analysis showed that TNM stage, NLR and BMI were independent prognostic factors. The new score system had a higher C-index, and the Calibration curves of the nomogram was reliable.

Conclusions

Based on the serum markers and other clinical indicators, we developed a precise model to predict the prognosis of patients with AEG after radical surgery. This score system can provide effective help for surgeons and patients.

\section{Background}

Adenocarcinomas of the esophagogastric junction (AEG) which located within $5 \mathrm{~cm}$ from esophagogastric junction(EGJ), was classified into 3 subgroups, type I AEG (adenocarcinoma of the distal esophagus) is the most prevalent type in Western countries, types II and III AEG are commoner than type I in Asia and are mostly treated as gastric cancer[1,2]. In recent years, the incidence of AEG is increasing rapidly in the world[3,4], surgery is considered the only means of curative treatment of AEG patients[5], however, the cancer recurrence rate and prognosis are still not optimistic even after radical resection.

Electronic gastroscope and Digestive tract angiography are important methods to diagnose AEG and judge the disease progression, however these techniques have high cost, risks, and inconvenience. At present, many studies are looking for noninvasive and sensitive biomarkers that can accurately predict the prognosis of AEG patients. Among them, commonly used serum markers for gastric cancer is carcinoembryonic antigen (CEA), which have been utilized for the early diagnosis of cancer and to screen for recurrences after surgery[6]. Additionally, there are also other serum index which can evaluate the 
prognosis of cancer, cancer-related systemic inflammatory response plays an important role in the progression and outcome of tumors [7,8], such as neutrophils to lymphocytes ratio (NLR), platelets to lymphocytes ratio (PLR). The presence of malnutrition can cause postoperative complications and poor prognosis [9], and nutritional marker are also having significant value for predicting survival. However, the relationship between these blood indexes and prognosis of AEG patients remains unclear.

To our best knowledge, there are few studies that had republished to access the prediction of inflammatory markers, nutritional markers and blood tumor markers for overall survival (OS) in AEG patients. Nomograms was a statistic model with a high reliability. In this study, we established a nomogram to explore the value of blood markers and then built a reliable model to predict overall survival after radical resection of $A E G$.

\section{Methods}

\subsection{Patients.}

We collected blood data and clinical data from AEG patients who were hospitalized in the First Affiliated Hospital of Anhui Medical University from January 2010 to January 2011. According the inclusion and exclusion criteria, patients were analyzed retrospectively during the research. The inclusion criteria included: 1) All patients were confirmed AEG by pathological diagnosis; 2) the surgery is definite and complete resection of cancer 3) these patients didn't have heart sickness or any important organs failures; 4) their peripheral blood tests were obtained within one week before surgery. The exclusion criteria included; 1 ) they had previous malignant tumors or various primary tumors; 2 ) they had accepted radiation treatment or chemo treatment previously before the treatment; 3 ) they had certain disease that could influences the counting of peripheral blood cells, such as infection; 4) they passed away within thirty days after the operation during the period of the follow-up. A cohort of 355 patients with AEG were included in this study.

\subsection{Data collection and follow-up.}

The data of patients' demographic and clinicopathological was gathered through the medical record room of our hospital, including age, gender, BMI, tumor size, differentiation grade and so on. The pathological tumor stage was categorized according to the AJCC 7th TNM staging system. The routine laboratory data were listed below: neutrophil, lymphocyte, platelet, prealbumin, albumin, hemoglobin and so on.

Peripheral blood tests were obtained within 1 week before surgery, we determine the following indexes (NLR, PLR, PNI). NLR got counted with the means of dividing the strict neutrophil counting with the strict lymphocyte counting, PLR was counted by dividing the strict platelet count by the strict lymphocyte count. PNI(prognostic nutritional index)was calculated using the following formula: serum albumin $(\mathrm{g} / \mathrm{L})$ $+5 \times$ total lymphocyte count $\left(10^{9} / \mathrm{L}\right)[10]$. NLR, PLR and PNI was grouped into low group and high group according to the Youden index [maximum (sensitivity + specificity-1)][11]. BMI was divided into 3 groups: 
$<18.5$ (low group), 18.5 to 24.9 (normal group), and $\geq 25$ (high group). CEA, CA199, albumin and prealbumin was grouped based on the normal value.

All Siewert type II/III of AEG patients underwent radical surgery with celiac and mediastinal lymphadenectomy. They accepted 4 to 6 cycles of first-line adjuvant combination chemotherapy after surgery with oxaliplatin plus 5-fluorouracil/leucovorin or a prodrug of 5-fluorouracil (capecitabine; CapeOX). The patients which were enrolled got prospective follow-up. Their follow-up date was obtained through telephones and outpatient visit. This behavior got carried out in normal intervals (each 90 days within two years after the surgery, each 180 days within the years of three-five, and once a year after five years). We followed up all the patients, 85 patients were excluded from the research, among them, 59 lost contact, 16 died of non-cancer-related deaths and 10 died within 30 days after surgery, in the end, 355 AEG patients were included in the final analysis of this study.

\subsection{Statistical analysis.}

Continuous variables were expressed as mean \pm standard deviation and they were analyzed by Student Ttest ; Categorical values were identified by quartile(P25, P75)and they were counted by Chi-square test or Fisher exact test. The multivariate and univariate surviving analysis were carried out using the Cox appropriate hazard pattern. Harrell's concordance index(C-index) was used in the nomogram for evaluating the model performance for prognosis of AEG patients. The Calibration curves was used to verify the accuracy of new score system in the nomogram. The whole data explanations got carried out applying SPSS app (16.0 version) and RStudio software (version 1.1.447-2009-2018; RStudio, Inc.) .

\section{Results}

\subsection{Baseline characteristics}

The baseline characteristics analysis of the 355 patients was expressed in the Table 1. Overall, 281 (79.1\%) patients were males and $74(20.9 \%)$ were females. The median age of patients was 65 years old (range, 29-85). The median follow-up month was 52 (range, 1.5-72). 


\begin{tabular}{|c|c|}
\hline characteristics & $\begin{array}{l}\text { Median(25th-75th percentile) } \\
\text { or no. }(\%)\end{array}$ \\
\hline \multicolumn{2}{|l|}{ gender } \\
\hline male & $281(79.1)$ \\
\hline female & $74(20.9)$ \\
\hline \multicolumn{2}{|l|}{ Age(year) } \\
\hline$<60$ & $246(69.3)$ \\
\hline$\geq 60$ & 168(30.7) \\
\hline \multicolumn{2}{|l|}{ Tumor size } \\
\hline$<5 \mathrm{~cm}$ & 164(69.1) \\
\hline$\geq 5 \mathrm{~cm}$ & 191(30.9) \\
\hline \multicolumn{2}{|l|}{ TNM stage } \\
\hline I- II & 154(43.4) \\
\hline III-IV & $201(56.6)$ \\
\hline \multicolumn{2}{|c|}{ Differentiation grade } \\
\hline low & $226(63.7)$ \\
\hline high & $129(36.3)$ \\
\hline \multicolumn{2}{|l|}{$\mathrm{BMI}(\mathrm{kg} / \mathrm{m} 2$ ) } \\
\hline$<18.5$ & $39(11.0)$ \\
\hline$\geq 18.5$ and $<25$ & $232(65.4)$ \\
\hline$\geq 25$ & $84(23.7)$ \\
\hline NLR & $2.28(1.57-3.12)$ \\
\hline $\begin{array}{l}\text { PLR } \\
\text { CEA } \\
\text { LT } \\
\text { AST }\end{array}$ & $\begin{array}{l}118.44(83.87-169.70) \\
3.20(1.60-8.20)\end{array}$ \\
\hline CA199 & $9.90(5.72-19.79)$ \\
\hline $\mathrm{PNI}$ & $49.53(45.80-53.25)$ \\
\hline albumin & $42.10(39.00-44.40)$ \\
\hline prealbumin & $209.00(165.00-255.00)$ \\
\hline
\end{tabular}




\begin{tabular}{|ll|}
\hline \multicolumn{2}{|l|}{ Table 1 Characteristics of the recruited patients } \\
\hline neutrophil & $3.37(2.57-4.51)$ \\
\hline platelet & $181.5(145-231)$ \\
\hline lymphocyte & $1.52(1.16-1.88)$ \\
\hline
\end{tabular}

3.2 Prognostic factors of the training cohort

Univariate risk factors of OS are shown in Table 2. The result showed that age, prealbumin, TNM, tumor size, histological type, CEA, PNI, PLR, NLR, BMI, hemoglobin and cancerous node were significant indicators, $P$ values of variables less than 0.05 in univariate analysis were includes in the multivariate analysis. The results showed that TNM, BMI and NLR were independent prognostic factors for OS (Table 3). 
Table 2

Univariate analysis of the AEG patients

\begin{tabular}{|llll|}
\hline Characteristics & $\boldsymbol{\beta}$ & HR(95\%Cl) & P value \\
\hline Gender(men/women) & 0.078 & $1.081(0.765,1.528)$ & 0.660 \\
\hline age & 0.019 & $1.019(1.002,1.037)$ & $0.031^{*}$ \\
\hline NLR & 0.176 & $1.193(1.112,1.280)$ & $<0.001^{*}$ \\
\hline Tumor size & 0.178 & $1.195(1.134,1.260)$ & $<0.001^{*}$ \\
\hline TNM stage & 1.042 & $2.836(2.046,3.930)$ & $<0.001^{\star}$ \\
\hline Histologic type & 0.390 & $1.477(1.086,2.009)$ & 0.013 \\
\hline CEA & 0.010 & $1.010(1.002,1.018)$ & 0.016 \\
\hline CA199 & 0.000 & $1.000(0.998,1.002)$ & 0.948 \\
\hline PNI & -0.034 & $0.966(0.940,0.993)$ & 0.013 \\
\hline PLR & 0.003 & $1.003(1.001,1.005)$ & 0.009 \\
\hline fibrinogen & 0.010 & $1.030(0.970,1.095)$ & 0.332 \\
\hline albumin & -0.289 & $0.479(0.557,1.008)$ & 0.056 \\
\hline prealbumin & -0.102 & $0.362(0.271,0.484)$ & $<0.001^{*}$ \\
\hline Surgery time & 0.017 & $1.017(0.755,1.369)$ & 0.912 \\
\hline BMI & -0.580 & $0.560(0.431,0.727)$ & $<0.001^{*}$ \\
\hline Cancerous node & 0.219 & $1.245(1.150,1.347)$ & $<0.001^{\star}$ \\
\hline hemoglobin & -0.006 & $0.994(0.988,1.000)$ & 0.033 \\
\hline
\end{tabular}


Table 3

Multivariate analysis of the AEG patients.

\begin{tabular}{|llll|}
\hline Characteristic & beta & HR(95\%Cl) & P value \\
\hline TNM & 0.827 & $2.286(1.236,4.227)$ & $0.008^{*}$ \\
\hline BMI & -0.470 & $0.625(0.413,0.946)$ & $0.026^{\star}$ \\
\hline CER & 1.092 & $2.979(1.565,5.674)$ & $0.001^{*}$ \\
\hline age & 0.008 & $1.008(0.997,1.019)$ & 0.143 \\
\hline Tumor size & 0.031 & $0.970(0.556,1.691)$ & 0.914 \\
\hline PNI & 0.143 & $1.154(0.651,2.045)$ & 0.624 \\
\hline PLR & 0.347 & $1.415(0.783,2.557)$ & 0.250 \\
\hline hemoglobin & 0.040 & $1.041(0.567,1.912)$ & 0.897 \\
\hline prealbumin & 0.197 & $0.821(0.479,1.408)$ & 0.474 \\
\hline Differentiation grade & 0.073 & $1.075(0.630,1.836)$ & 0.791 \\
\hline Cancerous node & 0.084 & $1.088(0.587,2.016)$ & 0.789 \\
\hline
\end{tabular}

3.3 Prognostic nomogram for survival

Based on the cox regression model, nomograms were constructed to predict overall survival of AEG(Fig. 1). In order to construct this nomogram, each subgroup variable was assigned a corresponding score, according to the specific situation of each $A E G$, a point system was used to assign a score ranging from 0 to 100 to each subgroup variable and the corresponding 3-, and 5-year OS rates was predicted, the nomogram scoring system was placed in Table 4.

Table 4

Nomogram Scoring System

\begin{tabular}{|llllll|}
\hline NLR & points & TNM & points & BMI & points \\
\hline low & 0 & low & 0 & high & 0 \\
high & 26 & high & 20 & normal & 58 \\
\hline & & & & high & 100 \\
\hline
\end{tabular}

3.4 Validation of the nomogram

Calibration curves were used to verify the performance of the model in predicting overall survival of AEG patients (Fig. 2, and Fig. 3). The C-index of the model was 0.697, indicating this model is reliable. 


\section{4.discussion}

Surgery is considered the only means of curative treatment of AEG patients. Due to the limitation of diagnostic techniques, it is often difficult to detect early AEG and lead to poor prognosis. At present, 5 years of survival rate is low. Therefore, in order to improve the prognosis of $A E G$, many scholars have made a lot of contributions. Studies have shown that elevated levels of markers may be associated with prognosis of AEG patients. Lymph node metastasis, tumor size, differentiation grade and TNM stage were defined as prognostic factors. However, these prognostic factors are difficult to judge before surgery, so the research on prognostic serum markers has been explored widely in recent years. To the best of our knowledge, this study is the first attempt to develop a prognostic nomogram which combined serum markers (including inflammatory markers, nutritional indexes and tumor markers) and clinicopathology characteristics for estimating the probability of 3-year and 5-year OS and showed a high accurate prediction for the prognosis of AEG.

Based on multivariate analysis, the results showed that TNM stage, NLR and BMI were independent prognostic factors for OS. So, we developed a nomogram of these markers, and the C-index was 0.673 , which indicated our new model is highly accurate in predicting the prognosis of AEG patients. Moreover, the Calibration curves of the nomogram is reliable.

In recent years, Nomograms showed high reliability for predicting tumor prognosis as a statistic model. Nomogram have better value for predicting prognosis in many cancer [12,13], this model has been identified as a new standard, and our study had got the same conclusion, it can be applied in the clinic, which can help surgeon to evaluate the prognosis of patients and take appropriate treatment.

Our nomogram contains three variables in which NLR and BMI are consistent with previous studies [14, 15]. Studies have suggested that systemic inflammation is an important factor affected the progression and long-term survival of cancer patients [16]. As simple and inexpensive clinical markers, NLR and PLR can reflect the state of inflammation, and are associated with poor prognosis of some cancer patients, but less reported in AEG. In this study, NLR was an independent risk factors, while PLR was not. The possible mechanism is that the systemic inflammation caused by malignant tumors can releases a large number of pro-inflammatory mediators, such as CRP, fibrinogen, VEGF, TGF-a, and so on. These factors stimulate tumor growth and metastasis [17], meanwhile the anti-tumor immune response of T cells and natural killer cells in the system may are surrounded by a number of neutrophils, this may decrease the opportunity to contact with tumor cells and have adverse effect on the prognosis of patients [18], besides, the monocyte also play important role in tumor progression [19], so NLR should be included in the regular assessment indexes of AEG patients.

As an independent prognostic indicator of tumor-related diseases, BMI has got more and more concern by researchers in recent years. BMI was related with the prognosis of breast carcinoma, non-small-cell lung cancer, and so on[20,21]. In this paper, we found that BMI was significantly correlated with the prognosis of AEG patients. The mechanism remains unclear. The AEG patients with low level of BMI may 
suffer poor nutritional status and immune function[22], this will have an adverse effect on the progression of the disease, so these patients may have short overall survival.

Our research has several potential limitations: First, this is a single-centered study, which has not enough cases to verify the results; secondly, the included patients who had undergone surgical resection for AEG could not behalf all AEG patients.

\section{Conclusion}

In summary, TNM stage, NLR and BMI levels were risk factors for the prognosis of AEG patients, and the novel nomogram model had reliable prognostic value for AEG patients.

\section{Abbreviations}

NLR: neutrophils to lymphocytes ratio PLR: platelets to lymphocytes ratio BMl: body mass index AEG: Adenocarcinomas of the esophagogastric junction EGJ: esophagogastric junction OS: overall survival PNI: prognostic nutritional index CEA囚carcinoembryonic antigen

\section{Declarations}

\section{Ethics approval and consent to participate}

This study was approved by the Institute Research Ethics Committee of The First Affiliated Hospital of Anhui Medical University, Hefei, China. Each patients signed the informed consent.

\section{Consent for publication}

Not applicable.

Availability of data and materials: The data used to support the findings of this study are available from the corresponding author upon request.

Competing interests: All authors declare no conflict of interest.

Funding: None.

Authors' contributions: LXZ designed this study, drafted this manuscript and revised this work. AMX and ZJW performed the study and participated in this work. All authors read and approved the final manuscript.

Acknowledgments: We thank Department of Gastrointestinal Surgery, the First Affiliated Hospital of Anhui Medical University for their contributions to our study.

\section{References}


[1] Fang WLखWu CW囚Chen JH囚et a1囚Esophagogastric junction ad- enocarcinoma according to Siewert classification in Taiwan $\square J] \rrbracket$ Ann Surg Oneol $₫ 2009 \otimes 16(12) \llbracket 3237-3244 \rrbracket$

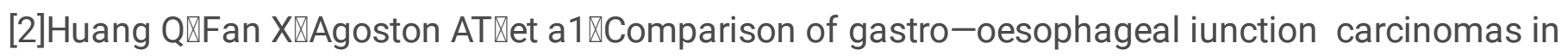

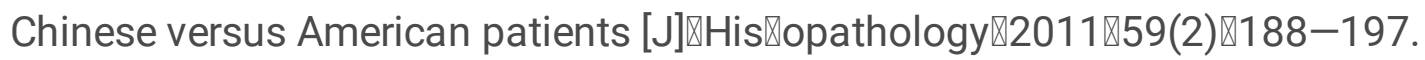

[3]. Zhao, J, Zhao, J, Du F, et al. Cardia and non-cardia gastric cancer have similar stage-for-stage prognoses after $\mathrm{R} 0$ resection: a largescale, multicenter study in China. J Gastrointest Surg.2016;20(4):700-7.

[4]. Carr JS, Zafar SF, Saba N, et al. Risk factors for rising incidence of esophageal and gastric cardia adenocarcinoma. J Gastrointest Cancer. 2013;44(2):143-51.

[5]. Saedi HS, Mansour-Ghanaei F, Joukar F, et al. Neoadjuvant chemoradiotherapy in non-cardia gastric cancer patients-does it improve survival? Asian Pac J Cancer Prev. 2014;15(20):8667-71.

[6] Deng K, Yang L, Hu B, Wu H, Zhu H, Tang C. The prognostic significance of pretreatment serum cea levels in gastric cancer: a meta-analysis including 14,651 patients. PLoS ONE. 2015; 4: e124151.

[7] Balkwill F, Mantovani A. Inflammation and cancer: back to Virchow. Lancet. 2001. 357(9255): 539-45.

[8] Hanahan D, Weinberg RA. Hallmarks of cancer: the next generation. Cell. 2011. 144(5): 646-74.

[9] Gomes de Lima KV, Maio R. Nutritional status, systemic inflammation and prognosis of patients with gastrointestinal cancer. Nutr Hosp2012; 27:707-14.

[10] Nozoe T, Ninomiya M, Maeda T, et al. Prognostic nutritional index: a tool to predict the biological aggressiveness of gastric carcinoma. Surg Today 2010;40:440-3.

[11] Youden WJ. Index for rating diagnostic tests. Cancer 1950;3:32-5.

[12] Zivanovic 0 , Jacks LM, lasonos A et al. A nomogram to predict postresection 5-year overall survival for patients with uterine leiomyosarcoma. Cancer 2012; 118: 660-9.

[13] lasonos A, Schrag D, Raj GV, Panageas KS. How to build and interpret a nomogram for cancer prognosis. J. Clin. Oncol. Off. J. Am. Soc. Clin.Oncol. 2008; 26: 1364-70.

[14]Zhang L, Su Y, Chen Z, et al. The prognostic value of preoperative inflammation-based prognostic scores and nutritional status for overall survival in resected patients with nonmetastatic Siewert type II/III adenocarcinoma of esophagogastric junction. Medicine (Baltimore). 2017 Jul;96(30):e7647.

[15]Huang L 1, Wei ZJ 1, Li TJ, et al. A prospective appraisal of preoperative body mass index in D2resected patients with non-metastatic gastric carcinoma and Siewert type II/III adenocarcinoma of esophagogastric junction: results from a large-scale cohort. Oncotarget.2017 Jul 12;8(40):68165-68179. 
[16] Roxburgh CS,Salmond JM,Horgan PG,et al.The relationship between the local and systemic inflammatory responses and survival in patients undergoing curative surgery for colon and rectal cancers[J].J Gastrointest Surg,2009,13:2011-2018.

[17] S.I. Grivennikov, F.R. Greten, M. Karin, Immunity, inflammation, and cancer, Cell140 (2010) 883-899.

[18] Smyth MJ, Dunn GP, Schreiber RD. Cancer immunosurveillance and immunoediting: the roles of immunity in suppressing tumor development and shaping tumor immunogenicity [J]. Adv Immunol, 2006,90:1-50.

[19] Evani SJ, Prabhu RG, Gnanaruban V, Finol EA, Ramasubramanian AK. Monocytes mediate metastatic breast tumor cell adhesion to endothelium under flow. FASEB journal. 2013; 27:3017-3029.

[20]Vernaci G , Dieci MV , Manfrin S, et al. BMI is an independent prognostic factor for late outcome in patients diagnosed with early breast cancer: A landmark survival analysis. Breast. 2019 Jul 20;47:77-84.

[21]Yendamuri S , Barbi J , Pabla S, et al. Body mass index (BMI) Influences the Salutary Effects of Metformin on Survival After Lobectomy for Stage I NSCLC. J Thorac Oncol. 2019 Aug 6. pii: S15560864(19)30650-1.

[22] Ji F, Liang Y, Fu S, et al. Prognostic value of combined preoperative prognostic nutritional index and body mass index in HCC after hepatectomy. HPB (Oxford) 2017;[Epub ahead of print].

\section{Figures}


Points

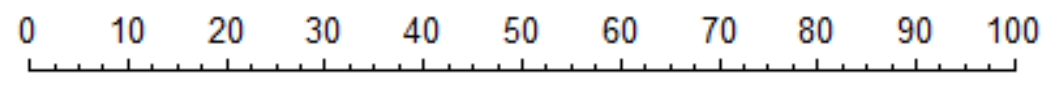

$\mathrm{BMI}$

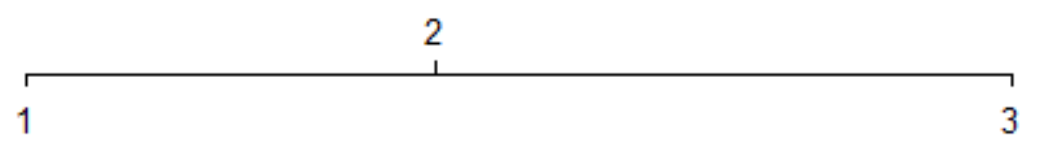

NLR

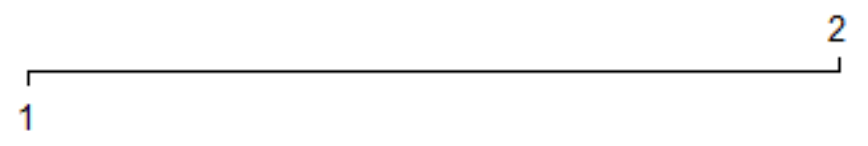

TNM

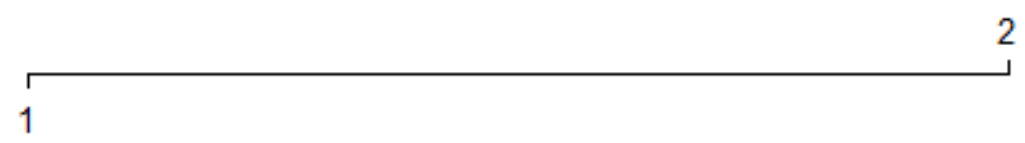

Total Points

$0 \begin{array}{llllll} & 1 & 1 & 1 & & \\ 0 & 100 & 150 & 200 & 250 & 300\end{array}$

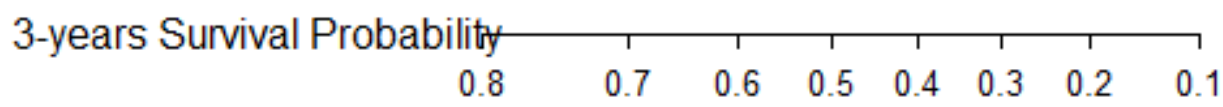

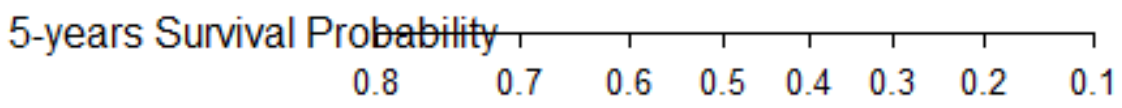

\section{Figure 1}

Nomogram for predicting overall survival after curative resection of gastric cancer 


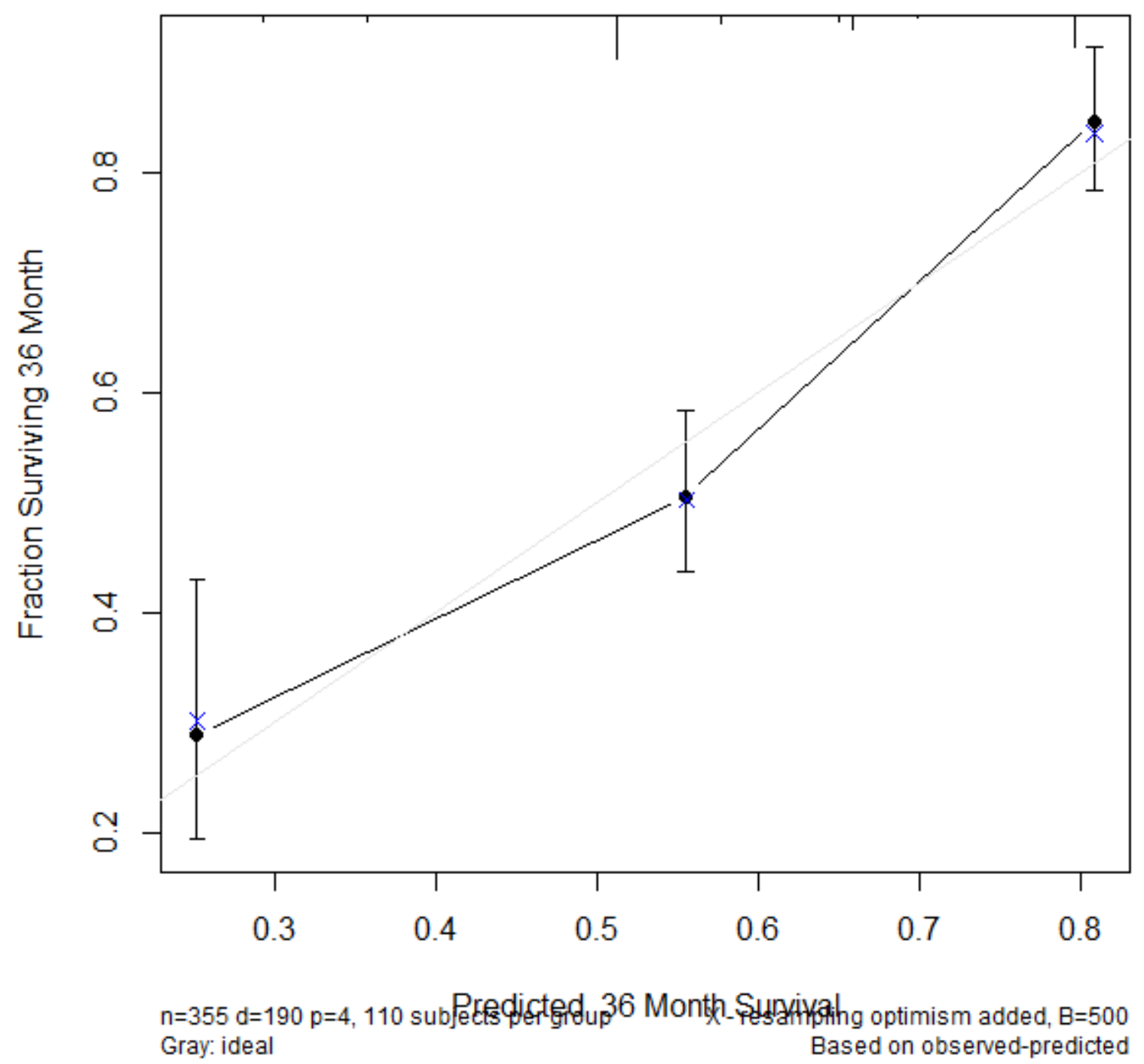

Figure 2

Calibration curves of the prognostic nomogram for 3-year overall survival 


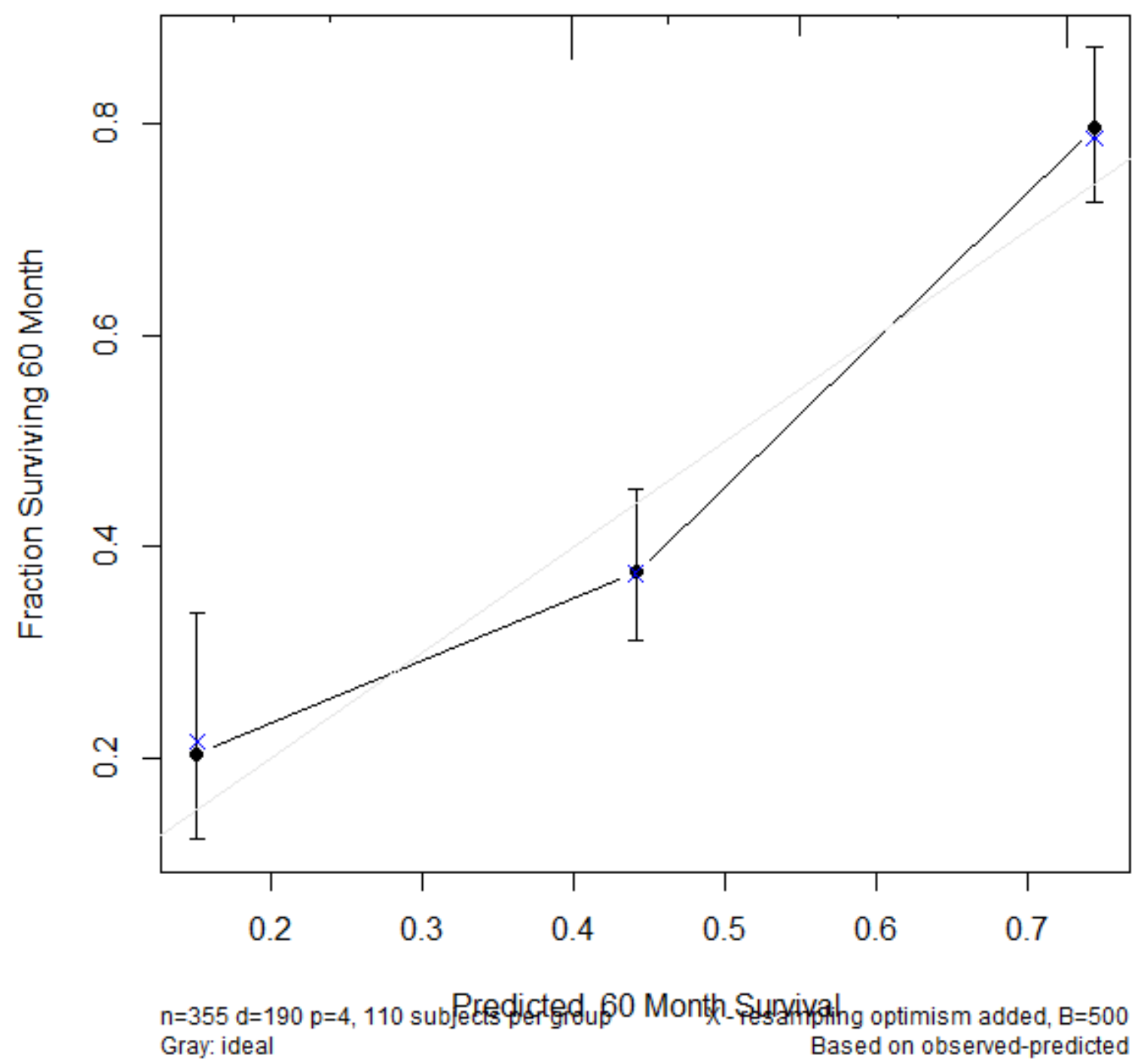

Figure 3

Calibration curves of the prognostic nomogram for 5-year overall survival 